\title{
CORRIGENDUM
}

\section{Cusps of Hilbert modular varieties}

\author{
BY D. B. McREYNOLDS \\ Math. Proc. Camb. Phil. Soc. (2008), doi: 10.1017/S0305004107001004;
}

First published online 18 February 2008

In this paper, the dates when the paper and its revised version were received, were inadvertently omitted. The missing information is:

Received 23 November 2006; revised 26 June 2007 